\title{
The role of sports games in young children sensory integrated training Li-feng Dong
}

Sports and Physics Department, Mianyang Normal University, 621000, China 273529824@qq.com

\begin{abstract}
Children sensory integration training needs for training through a variety of ways the senses of children, there are flexible in the training mode, and sports games in its category and set also has the same advantages. The two of organic integration easier implementation of sensory integration training. The sports games into children sensory integration training to achieve the purpose of training, for the development of the ability and the children have a very good role.
\end{abstract}

Keywords: Early childhood education, sports games, sensory integration training

\section{体育游戏在幼儿感觉统合训练中的作用 \\ 董理峰 \\ 绵阳师范学院体育与健康教育学院, 绵阳, 四川, 中国}

273529824@qq.com

中文摘要: 幼儿感觉统合训练需要通过多种 方式对幼儿的各项感官进行训练, 在训练的 方式上有着灵活多样的特点, 而体育游戏其 在种类与设置上也具备着同样的优点。因此 将二者进行有机的融合更容易实现幼儿感 觉统合的训练。将体育游戏融入到幼儿感觉 统合训练中去, 对于训练目的的实现以及幼 儿能力的培养等都有着很好的作用。

关键词: 幼儿教育; 体育游戏; 感觉统合训 练

\section{1. 前言}

幼儿教育是我国教育教学的基础, 也是 教育教学的重中之重, 其不仅对学生今后的 学习有着一定的影响, 同时对学生的心理建
设, 性格发展等都有着巨大的影响作用。因 此在进行幼儿教育的过程中如何有效的对 幼儿进行感觉统合训练, 促进幼儿的健康成 长, 是广大幼儿教育工作者以及家长共同关 注的问题。体育游戏在幼儿教育中占有着一 定的地位, 在对幼儿教育的过程中开展体育 游戏, 符合幼儿的天性, 有益于幼儿教学的 展开。而且通过近几年对体育游戏的研究与 探索我们发现将体育游戏与幼儿感觉统合 训练相结合, 可以有效的提高幼儿感觉统合 训练的质量, 更加有益于幼儿的成长与发 展。

\section{2. 幼儿感觉统合训练概述}

\section{1 幼儿感觉统合训练的概念}

英国知名生理学者C.S. 谢林顿提出了 感觉统合的概念。在这个基础上, Jean A 
ryes 这位临床心理学家系统性的提出了感 觉统合的理论与感觉统合失调的原因及治 疗方法。根据感觉统合的相关研究及经典理 论, 感觉统合是大脑在环境适应过程中对外 界环境信息所做出的一系列正确的应答。从 已有研究成果来看, 对于感觉统合的发生机 理, 不同学者有着不同的认识与理解。其中, Jean A ryes从信息传递过程的角度提出了 自己的观点, 认为情感、思维、记忆、注意 等心理活动的过程及状态实际上并不是由 大脑皮层的某个区域独立完成的, 而是一种 信号传递过程, 是一类信息在不同时间和空 间以某种信号的形式存储在大脑皮层, 再在 大脑皮层的相应功能区域进行联系和统一 解释的结果。后来, 这种观点被称为信息理 论学说, 得到了学界很多学者的认同。进一 步的研究提出, 人类所具有的推理、注意、 思维和记忆等方面的能力, 以及相应的心理 行为、情感、认知过程, 其发生的机制主要 是大脑对感觉信息通过联系、比较、控制、 选择和增强, 对自身不同区域的信息加以整 合的过程。对发生机制的深入认识发现, 感 觉信息起源于外界刺激, 当来自外界的气 味、声音、光、温度、重力、触压等刺激达 到人体感知的强度时, 大脑就会对感知到的 刺激信息进行综合处理和反复分析, 最终做 出正确的决策。当然, 这里所说感觉统合是 大脑对外界环境信息的一系列正确应答, 其 重要前提是心理行为必须是正常的。否则, 身体、大脑与环境三者之间便无法得以协 调, 这就是缺少感觉统合导致的, 也被称为 感觉统合失调。事实证明, 感觉统合能力是 身体、情感、社会性以及认识能力发展的必 要基础。如果没有感觉统合, 身体与大脑就 不能实现健康发展, 儿童就不能完成正常的 学习过程和成长过程。针对这个问题, 感觉 统合理论明确指出了儿童的大脑有着非常 高的可塑性与可发展性, 通常儿童感觉统合 能力逐渐形成于 3 岁以内, 并且在儿童日后 的生活和成长中不断的得以发展、重构以及 加强, 并进一步提出了人脑的功能发展不足 以及学习障碍问题是可以通过一些训练方 法进行矫正的, 这些训练方法被称为感觉统 合训练。感觉统合训练促使人体不同部分的
神经系统更好地与整体作用相互协调, 实现 环境与个体的顺利接触。

幼儿感觉统合训练这一概念来源于国 外, 其主要的目的就在于通过轻松愉快的教 学方式, 引导幼儿向着积极向上的方向发 展, 开发幼儿智力, 提高幼儿体质。这种训 练模式在不断研究与发展的过程中, 进行了 一定的改革与完善, 就目前而言其已经具备 了一定的推广以及普及的基础。目前我国幼 儿教育已经开始引入幼儿感觉统合训练希 望通过幼儿感觉统合训练的方式, 提高幼儿 的培育及教育水平, 为幼儿今后的成长打好 基础, 具体而言在我国幼儿教育中幼儿感觉 统合训练包含以下几个方面的内容:

智力的开发。智力开发一直是教育教学工作 的重要组成部分, 如何有效激发学生的潜 力, 促进学生的进步是广大教育工作者们共 同努力的方向。幼儿正处于人生发育的最佳 时期, 在幼儿阶段对其进行智力的开发, 将 对其今后智力的发展起到正向的影响, 有益 于其智商甚至是情商的提高。（2）身体素 质的提升。幼儿的身体相对比较脆弱, 提高 幼儿的身体素质将在很大程度上可以有效 减少幼儿成长过程中的阻碍, 有利于幼儿的 健康发展。因此在我国幼儿教育的过程中进 行感觉统合训练, 提高幼儿身体素质是其重 要的任务之一。（3）行为习惯的修正。幼 儿虽然在心智的发展上仍处于懵懂阶段, 但 是由于受到社会, 以及家庭等方面的影响, 其经常会表现出一些个性化的行为以及习 惯, 在这些行为习惯中有些对于幼儿的成长 会造成一定的不良影响, 因此在进行幼儿感 觉统合训练的过程中, 教师一般对通过训练 的方式对这些行为以及习惯进行修正, 培养 幼儿从小建立起正确的行为习惯。

\section{2 幼儿感觉统合训练的特点}

幼儿感觉统合训练与一般的幼儿教学 方法存在着明显的不同, 其在对幼儿进行教 育教学的过程中凸显出了大量的特点以及 优势, 可以更加有利于幼儿的成长发育, 具 体来说幼儿感觉统合训练具有以下几个方 面的特点: (1) 侧重科学合理。幼儿感觉 统合训练是一种基于科学理论认知发展起 来的教育模式, 其在进行教育教学的过程中 更加的侧重了教育教学的科学性, 合理性, 
在对幼儿进行感觉统合培训的过程中, 幼儿 教师或是感觉统合训练的执行者, 应保持科 学的态度, 对幼儿的训练采取循序渐进的方 式进行, 以保障幼儿可以有效的接受训练并 取得理想的效果。（2）注重个性的发展。 每一个幼儿都是一个独立的个体, 虽然其在 意识以及表达能力上还存在着一定的欠缺, 但在其成长的过程中仍有着个性化的需求, 也表现出了其作为个体与众不同的特点, 因 此在进行幼儿感觉统合培训的过程中注重 幼儿个性的发展, 成为了其主要的特点之 一。这一特点有利于帮助幼儿自由发展, 对 于其性格的塑造有着重要的帮助作用。(3) 训练方式的灵活多样。幼儿感觉统合训练主 要是利用幼儿的各项感官对其进行有效的 训练, 达到帮助幼儿健康成长的目的。因此 针对幼儿不同的感官, 教师一般都会安排有 针对性的培训, 以达到最佳的效果, 例如, 在对幼儿视觉进行训练时一般会进行色彩 绘画的教学, 而进行听觉的训练时则多会借 助音乐的帮助。因此幼儿感觉统合训练在训 练方式上突出表现除了灵活多样的特点。

\section{3 幼儿感觉统合训练的原则}

幼儿教学是一项严谨而神圣的工作, 在对幼儿进行教育教学的过程中, 教育工作 者以及家长都要对幼儿的接受能了以及幼 儿的心理予以重视, 因此在进行幼儿感觉统 合训练的过程中, 教师也应时刻保持小心谨 慎的态度, 坚持幼儿感觉统合训练的各项原 则, 保障幼儿感觉统合训练顺利进行。具体 来说幼儿感觉统合训练的原则包括以下几 个方面: (1) 坚持以游戏为本。开展游戏 教学是幼儿感觉统合训练的主要内容之一, 其从幼儿的天性出发, 利用幼儿参与游戏的 积极性, 通过游戏的方式对幼儿进行感觉统 合训练。这种训练的方式迎合了幼儿发育成 长的特性, 是进行幼儿感觉统合训练过程中 应该坚持的基本原则。（2）从幼儿的兴趣 出发。幼儿在成长的过程中, 对周围世界有 着巨大的好奇心以及探索欲望, 其很容易受 到外界因素的影响, 产生各种兴趣以及爱 好。因此在对幼儿进行感觉统合训练的过程 中教师应把握幼儿的兴趣发展, 提高训练方 法的趣味性, 探索性, 让幼儿可以积极的参 与其中并在不断探索的规程中完成各项训
练任务。（3）严格保障幼儿安全。幼儿在 实际的生活中几乎没有自我保护的能力, 因 此在对幼儿进行教育教学的过程中严格保 障幼儿教学的安全是关键。这不仅是幼儿感 觉统合训练的原则之一同时也是所有幼儿 教育教学模式都应遵守的首要原则。 坚持训练的发展与完善。幼儿感觉统合训练 虽然目前已经引入到了幼儿的教育教学中, 但是其自身仍然存在着一定的问题, 并有着 巨大的发展潜力。因此在对幼儿进行感觉统 合训练的过程中幼儿教师应用发展的眼光 看问题, 坚持训练的发展与完善, 促进幼儿 感觉统合训练水平的不断提升。

\section{3. 体育游戏在幼儿感觉统合训练中的作用}

\section{1 促进幼儿的身体发育, 提高幼儿的身 体素质}

尽管感觉统合失调尽管不会随着儿童 的成长而自动消失, 但是却会随着刺激练习 的逐步加强和身体发育的不断完善而得到 改善, 儿童年龄逐渐增长, 肌肉控制能力逐 步增强, 神经系统日趋成熟, 儿童的感觉统 合便会逐步协调, 感觉统合失调症状得以缓 解。因此, 幼儿阶段是感觉统合失调症治疗 的最佳时期, 也就是说幼儿教育阶段时期对 感觉统合失调的幼儿而言进行系统训练是 尤为重要的。将训练有效的方式与体育游戏 相互融合, 使孩子们能够在自然、轻松快乐 以及积极主动的教学情境当中尽情的学习 和玩要, 加强手眼协调、本体感觉、平衡觉 以及触觉等能力的训练, 从而使得多种感知 信息能够输入到学生的大脑当中, 不断的促 进学生大脑综合的分析信息, 同时加强身体 和大脑二者间的协调反应, 帮助孩子的感觉 运动可以始终朝着正常的方向不断的发展, 最终达到开发幼儿的身心潜能、培养良好行 为、增强身体体质以及促进智力发展的目 的。

身体发育是幼儿成长中的重要问题之 一, 关系着幼儿今后的身体素质以及工作生 活。因此大多数的家长以及幼儿教师都对幼 儿的身体发展情况给予极大的关注。而对幼 儿进行感觉统合训练的过程中, 大量的引入 体育游戏, 可以增加幼儿的运动量, 在一定 
程度上对幼儿的身体发育可以起到促进作 用, 可以有效的提高幼儿的身体素质, 为其 今后身体的成长奠定良好的基础。

\section{2 帮助幼儿融入社会群体, 培养学生集 体意识}

人类是社会型动物, 其在生存与发展 的过程中离不开人与人之间的交流, 因此加 强幼儿对群体的适应性, 对于幼儿今后的发 展有着良性的引导。通过教育学家进行研究 证明, 组织学生进行群体性活动, 增加学生 之间的互动, 对学生今后融入社会将有很多 的帮助作用。一般而言在进行幼儿感觉统合 训练的过程中教师会有侧重的组织幼儿进 行团体性的体育游戏活动, 让幼儿在游戏的 过程中融入到群体生活中去, 加强同龄人之 间的交流，培养学生的集体意识。

\section{3 有益于学生智力的开发, 能力的提升}

体育游戏在幼儿感觉会统合训练中进 行应用后其不仅对幼儿的身体素质给予了 提升, 同时对于开发幼儿智力也有着不可忽 视的作用。就目前来看, 在进行体育游戏的 过程中, 幼儿会积极主动的进行自主性的思 考, 并通过自己的方式对游戏的内容以及游 戏的规则等产生认识。例如, 在进行传统游 戏丢手绢的过程中, 幼儿为了不被其他同学 抓住就会对游戏规则以及游戏内容进行思 考, 选取其自己认为最有力的方式进行手绢 的投放, 在这一选择的过程中, 其通过一系 列肢体的活动, 作用于思维的发散, 充分的 锻炼了大脑的判定力以及逻辑思维能力。让 幼儿在愉悦的氛围中, 开发个人智力, 提升 个人能力。

\section{4 保障幼儿心理健康, 促进健全人格的 生产}

心理健康问题是现代社会急需解决的 问题, 现代研究证明, 当前社会中大部分的 人都存在着或多或少的心理问题, 而这其中 青少年占有着巨大的比例。因此为了让幼儿 在今后的成长中避免受负面情绪的不良影 响, 保障幼儿的健康成长, 对幼儿的心理健 康予以一定的重视是十分有必要的。幼儿感 觉统合训练在这一方面具有突出的优势, 其 通过体育游戏的方式, 将挫折教育, 团体教
育等多种教育思想渗透其中, 让幼儿在进行 训练的过程中, 形成积极正向的人身态度。 有利于其今后心理的健康以及健全人格的 形成。

以往人们对于健康的认识通常局限于 “没有疾病”，而在 “三维健康观” 的观点 提出后, 人们便更进一步的对 “健康” 广度 进行了认识。但是就健康的深度而言, 比如 感觉统合失调, 尚且未得到充分的关注和重 视，从所搜集到的体育专著及体育教材当 中, 除了极少书籍对感觉统合失调进行了阐 述和分析以外, 其它体育专著及教材都没有 涉及到这方面的内容。所以, 加深理解和认 识 “健康” 的思想, 并且积极的运用先进的 教学手段加以实行, 只有这样才可以真正的 对 “健康第一” 的教育思想加以贯彻落实。 就感觉统合失调问题而言, 神经尤其是中枢 神经, 可以说是人体感觉统合不可或缺的基 础, 这便从根本上决定了感觉统合失调原因 的探寻应当首先从研究中枢神经着手。由于 幼儿感觉统合是中枢神经多个区域相互协 同作用的结果，所以，感觉神经中枢的不同 区域间是密切联系和合作的有机整体, 无论 哪种中枢神经和感觉外围神经受损或者缺 陷, 均会导致统合神经的协作和连结途经发 生中断而造成感觉统合失调。大量的实践教 学充分的表明, 在幼儿教育阶段的体育课程 教学或体育游戏活动当中进行感觉统合训 练, 可以对幼儿教育阶段孩子的感觉统合能 力的发展产生积极的促进作用, 因而, 将幼 儿教育阶段体育课程或体育游戏活动与幼 儿感觉统合训练进行融合是非常重要的, 也 是非常必要的。

\section{4. 体育游戏在幼儿感觉统合训练中的应用}

\section{1 适当开展体育竞技, 激发学生潜力}

将体育游戏融入到幼儿感觉统合训练 中去。可以更好的实现幼儿感觉统合的训 练。而在体育游戏的过程中, 组织学生进行 体育游戏竞赛更是可以对学生的各项能力 进行激发与促进。具体来将教师可以通过以 下几种方式组织学生进行体育游戏竞赛:

(1) 亲子组合共同参赛。幼儿由于年龄合 小, 因此其没有能力独自进行某些体育游戏 
的竞赛活动。鉴于此种状况, 教师可以在亲 子日组织家长与幼儿共同参加比赛, 这样不 仅有益于幼儿各项能力的培养, 更在一定程 度上增加了家长与孩子之间的交流, 有利于 培养良好的亲子关系。(2) 选择相对简单 的比赛项目。教师在进行体育游戏的选择与 安排的过程中要充分的考虑到幼儿年龄, 教 学环境等多方面的因素, 以保障体育游戏的 顺利展开。在组织幼儿进行体育游戏竞赛的 过程中, 教师更应加大对游戏选择的重视, 尽量选择对幼儿有吸引力并且容易完成的 体育游戏进行竞技。

\section{2 开展样式多样的体育游戏, 提高幼儿 的综合素质}

幼儿感觉统合训练需要通过多种方式 对幼儿的各项感官进行训练, 因此其在训练 的方式上有着灵活多样的特点, 而体育游戏 其在种类与设置上也具备着同样的优点。因 此将二者进行有机的融合更容易实现幼儿 感觉统合的训练。具体来说在教师在利用体 育游戏进行幼儿感觉统合训练的过程中应 从以下几个方面入手: (1) 开展样式多样 的体育游戏。体育游戏样式繁多, 并且在进 行游戏的过程中教师还可以根据幼儿的情 况进行灵活的调整。因此在进行幼儿感觉统 合训练的过程中, 教师一般会准备大量的体 育游戏方案, 并针对要训练的内容进行合理 的选择, 以达到幼儿感觉统合训练的目的。

（2）营造适当的游戏氛围。幼儿的注意力 一般比较容易分散, 外界的任何影响因素都 可能引起其的注意, 导致感觉统合训练的中 断甚至失败。因此教师在组织学生进行体育 游戏的过程中应注意营造适当的游戏氛围, 将幼儿引入到游戏的氛围中去, 提高幼儿的 注意力, 促进幼儿增和素质的提高。

\section{3 进行团体游戏的推广, 培养学生团队 协作}

体育游戏可以是个人的也可以是团体 的, 在对幼儿进行感觉统合训练的过程中不 仅要针对幼儿个人进行有针对的训练, 同时 也要适时的组织幼儿进行团体的活动, 以帮
助幼儿适应集体生活, 提高交流能力。具体 来讲在进行团体游戏的过程中教师应做到 以下几个方面:（1）进行团体游戏的选择, 协调游戏的进行。团体游戏的种类有很多, 在进行游戏的选择过程中, 教师应选择肢体 冲突小, 安全系数高的游戏进行, 避免选择 足球, 篮球等具有危险性的运动。可以组织 学生进行一些传统游戏项目如丢手绢, 捉迷 藏等。或者教师还可以根据具体的教学情境 制定一些创新型的游戏。除此之外, 在进行 游戏的过程中, 教师也要提高注意, 对幼儿 的行为给予全面的关注, 协调游戏的进行。

（2）促进幼儿之间互相协作。就我国当前 的国情来看, 独生子女居多, 大对数的幼儿 在成长的过程中缺乏与同龄人沟通与玩要 的经验, 造成其在日后的生活中难以融入社 会生活, 出现一定的沟通障碍等。因此为了 有效的解决这一问题, 幼儿感觉统合训练在 训练的过程中通过进行团体体育游戏的方 式, 促进幼儿之间的团结协作, 通过加强幼 儿间交流的方式，让幼儿对集体，对合作产 生印象。

\section{基金项目:}

本文为四川省农村幼儿教育研究中心 资助项目（项目编号：NYJ20130607）。

\section{References}

[1] Xiao Jianzhong, Sensory integration training of primary school physical education teaching, Journal of physical education, vol.19, pp. 93-97, 2012.

[2] Chi Liyan, Research on sports practice infant school education in China from the sensory integration training, Elementary Education, vol.12, pp. 113-117, 2012.

[3] Cai Jiyun, The feasibility study of sensory integration training applied in infant sports, Journal of Hebei Union University (Medical Science Edition), vol. 15, pp.591-592, 2013. 